\title{
Cardiovascular drugs and mortality in hypertensive patients with atrial fibrillation: evidence of efficacy for a global approach
}

\author{
Arrigo FG Cicero, Giuseppe Derosa and Claudio Borghi \\ Hypertension Research (2014) 37, 492-493; doi:10.1038/hr.2014.64; published online 13 March 2014
}

\begin{abstract}
$\mathrm{A}^{\mathrm{t}}$ trial fibrillation (AF) is the most common type of arrhythmia encountered in clinical practice, and nearly one in four individuals develops this condition within their lifetime. As a result, AF carries a significant burden in terms of both morbidity and mortality. Costs and hospitalizations attributable to AF have increased markedly in recent decades, with an estimated cost greater than $1 \%$ of all health care expenditure in Western countries, and this burden is expected to increase in the future due to aging of the population. ${ }^{1}$ Thus, each new strategy aimed at improving the prognosis of $\mathrm{AF}$ patients must be carefully considered. Current treatment for AF involves either rate or rhythm control, although both approaches have demonstrated associated risks. Rate control necessitates anticoagulation, which can cause life-threatening bleeding, whereas rhythm control carries a poor side-effect profile that may lead to greater mortality and may not completely eliminate the need for anticoagulation.
\end{abstract}

In a very large study, Carlsson et al. ${ }^{2}$ showed that hypertensive patients complicated with AF presented significantly lower relative mortality risks after receiving statins, nonselective beta-blockers and angiotensin receptor blockers compared with patients who were not taking these drugs. By contrast, treatment with loop diuretics was associated with increased mortality.

AFG Cicero and C Borghi are at Department of Medicine and Surgical Sciences, University of Bologna, Bologna, Italy; G Derosa is at Department of Internal Medicine and Therapeutics, University of Pavia, Pavia, Italy

E-mail: arrigo.cicero@unibo.it
Therefore,these interesting data merit further evaluation and discussion.

Preliminary data suggest that blockade of the renin-angiotensin system may protect patients from AF-related complications. However, in the large ACTIVE-I trial, which included 9016 patients who were followed for a mean period of 4.1 years, irbesartan was not found to reduce cardiovascular events in patients with $\mathrm{AF}^{3}$ Nevertheless, it must be noted that the control group in this study achieved similar blood pressure control with the use of a number of efficacious antihypertensive treatments, which could have complicated the interpretation of the data obtained. Similarly, in the study of Carlsson et al., ${ }^{2}$ patients who obtained optimal protection from AF-related complications were those who received greater numbers of antihypertensive drugs and therefore achieved more complete control over the pathophysiological mechanisms underlining hypertension and the related consequences.

With regard to beta-blockers, these drugs are known to significantly improve heart rate control. However, a recent meta-analysis including 10 studies with 7867 patients with $\mathrm{AF}$ showed that rate-control strategies $v s$. rhythm-control strategies were similar with regard to in-hospital mortality, rates of stroke, systemic embolism, worsening heart failure, myocardial infarction and bleeding. Furthermore, the rates of rehospitalization were much lower for patients who received a rate-control strategy $(P=0.007)$. On the other hand, an exploratory analysis in patients younger than 65 years of age found that a rhythm-control strategy was superior to rate control in the prevention of all-cause mortality $(P=0.0007) .{ }^{4}$ These data are partially in agreement with the findings of Carlsson et al; ${ }^{2}$ however, it is very difficult to distinguish the antihypertensive and antiischemic actions of beta-blockers from an eventual specific effect on AF-related complications.

In another meta-analysis including 23577 patients, statin therapy was significantly associated with a decreased risk of AF compared with control treatment (odds ratio $(\mathrm{OR})=0.49, \quad 95 \% \quad$ confidence interval $(\mathrm{CI})=0.37-0.65 ; P<0.001)$. In particular, a beneficial effect was observed in the atorvastatin subgroup and the simvastatin subgroup, but not in the pravastatin subgroup or the rosuvastatin subgroup. In addition, the benefit of statin therapy appeared to be more pronounced in secondary prevention $(\mathrm{OR}=0.34,95 \% \mathrm{CI}=0.18-0.64 ; P<0.001)$ than in primary prevention $(\mathrm{OR}=0.54,95 \%$ $\mathrm{CI}=0.40-0.74 ; P<0.001) .{ }^{5}$ However, there is still no specific evidence showing that statins can reduce mortality in AF patients beyond their anti-ischemic effect and their overall effect on decreasing cardiovascular disease risk. In addition, in the study of Carlsson et al., ${ }^{2}$ it was impossible to distinguish the cardiovascular disease-preventing activity of statins from an eventual specific effect on AFcomplications.

In fact, a prospective cohort of 23928 participants who were followed for a mean of 6.9 years (median 4.5 years) clearly showed that $\mathrm{AF}$ was associated with an approximate twofold increased risk of myocardial infarction (hazard ratio $=1.96,95 \% \mathrm{CI}=1.52$ 2.52). Moreover, this association remained significant (hazard ratio $=1.70, \quad 95 \%$ $\mathrm{CI}=1.26-2.30)$ after further adjustment for 
OR

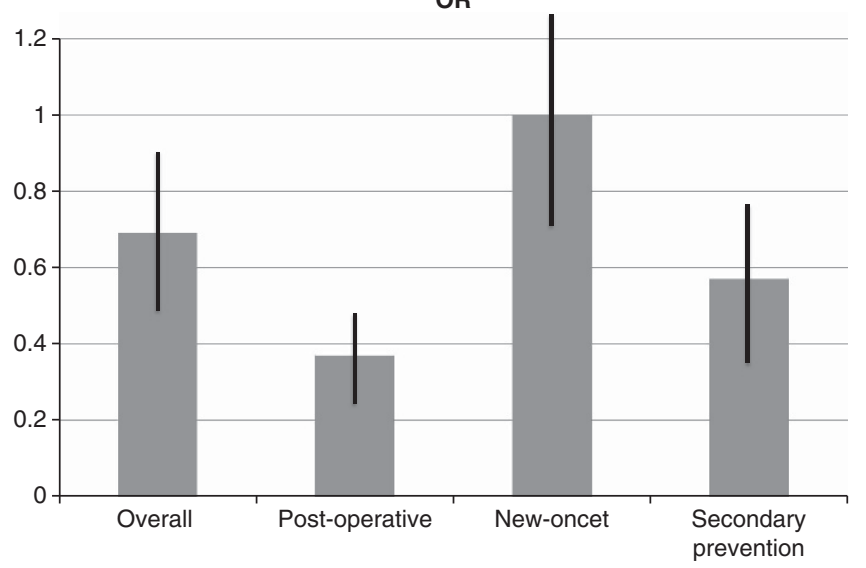

Figure 1 Statin treatment and risk to develop atrial fibrillation or recidivism in different settings (from Mills et al. ${ }^{7}$ )

total cholesterol, high-density lipoprotein cholesterol, smoking status, systolic blood pressure, blood pressure-lowering drugs, body mass index, diabetes, warfarin use, aspirin use, statin use, history of stroke and vascular disease, estimated glomerular filtration rate, albumin to creatinine ratio and C-reactive protein level. ${ }^{6}$ Because the preventive effect of statins is proportional to the baseline global cardiovascular disease risk, ${ }^{7}$ patients at highest risk due to concomitant AF may experience a larger preventive advantage following statin use. On the other hand, statins may exert a positive impact on $\mathrm{AF}$ prevention and $\mathrm{AF}$ recidivism (Figure 1), ${ }^{8}$ suggesting a potentially relevant protective effect on atrial conduction fibers, and consequently on the clinical outcomes of patients.

There is also an increasing body of evidence suggesting that an inexpensive marker frequently increased in AF patients, serum uric acid, could be associated with myocardial function and other cardiovascular risk factors $^{9}$ as well as an increased risk of mortality. ${ }^{10}$ In particular, data from a metaanalysis showed that elevated serum uric acid levels were significantly associated with an increased risk of all-cause mortality (risk ratio $=1.24, \quad 95 \% \quad \mathrm{CI}=1.09-1.42) \quad$ and cardiovascular mortality (risk ratio $=1.37$, $95 \% \mathrm{CI}=1.19-1.57)$. Serum uric acid levels may also be positively or negatively influenced by other preventive drugs (e.g., modulators of the renin-angiotensin system and statins are tendentially associated with an improvement in clinical parameters, whereas diuretics and beta-blockers present a negative association). Therefore, the serum uric acid level and other cardiovascular risk factors modulated by concomitant therapy should be taken into account when adequately interpreting the study of Carlsson et al. ${ }^{2}$

Finally, regarding the increased mortality observed among loop diuretic-treated patients in the study of Carlsson et al., ${ }^{2}$ it should be noted that the authors did not adjust the data for the chronic heart failure class, and it is possible that patients with more severe heart failure were also treated with loop diuretics.

Nevertheless, on the basis of the available evidence, it seems that a global approach to the cardiovascular health of AF patients, on the basis of intense and complete treatment with preventive drugs shown to be effective and assessment of the most relevant cardiovascular risk factors, could have a positive effect on the total mortality, beyond the effect on the single-risk factors treated.

\section{CONFLICT OF INTEREST}

The authors declare no conflict of interest.

1 Ball J, Carrington MJ, McMurray JJ, Stewart S. Atrial fibrillation: profile and burden of an evolving epidemic in the 21st century. Int J Cardiol 2013; 167: 1807-1824.

2 Carlsson AC, Wändell P, Sundquist K, Johansson SE, Sundquist J. Effects of prescribed antihypertensives and other cardiovascular drugs on mortality in patients with atrial fibrillation and hypertension: a cohort study from Sweden. Hypertens Res 2014; 37: 553-559.

3 ACTIVE I Investigators, Yusuf S, Healey JS, Pogue J, Chrolavicius S, Flather M, Hart RG, Hohnloser SH, Joyner CD, Pfeffer MA, Connolly SJ. Irbesartan in patients with atrial fibrillation. New Engl J Med 2011; 364: 928-938.

4 Chatterjee S, Sardar P, Lichstein E, Mukherjee D, Aikat S.. Pharmacologic rate versus rhythm-control strategies in atrial fibrillation: an updated comprehensive review and meta-analysis. Pacing Clin Electrophysiol 2013; 36: 122-133.

5 Fang WT, Li HJ, Zhang H, Jiang S. The role of statin therapy in the prevention of atrial fibrillation: a metaanalysis of randomized controlled trials. $\mathrm{Br} \mathrm{J} \mathrm{Clin}$ Pharmacol 2012; 74: 744-756.

6 Soliman EZ, Safford MM, Muntner P, Khodneva $Y$, Dawood FZ, Zakai NA, Thacker EL, Judd S, Howard VJ, Howard G, Herrington DM, Cushman M. Atrial fibrillation and the risk of myocardial infarction. JAMA Intern Med 2013; 174: 107-114.

7 Mills EJ, Wu P, Chong G, Ghement I, Singh S, AkI EA, Eyawo O, Guyatt G, Berwanger O, Briel M. Efficacy and safety of statin treatment for cardiovascular disease: a network meta-analysis of 170,255 patients from 76 randomized trials. QJM 2011; 104: 109-124.

8 Fauchier L, Clementy N, Babuty D. Statin therapy and atrial fibrillation: systematic review and updated metaanalysis of published randomized controlled trials. Curr Opin Cardiol 2013; 28: 7-18.

9 Cicero AF, Rosticci M, Parini A, Baronio C, D'Addato S, Borghi C. Serum uric acid is inversely proportional to estimated stroke volume and cardiac output in a large sample of pharmacologically untreated subjects: data from the Brisighella Heart Study. Intern Emerg Med (e-pub ahead of print 9 November 2013; doi:10.1007/ s11739-013-1016-9).

10 Zhao G, Huang L, Song M, Song Y. Baseline serum uric acid level as a predictor of cardiovascular disease related mortality and all-cause mortality: a meta-analysis of prospective studies. Atherosclerosis 2013; 231: 61-68. 